\title{
Maturação Funcional da Retina em Bebês Prematuros
}

\author{
Adriana Berezovsky ${ }^{1}$ \\ Universidade Federal de São Paulo - UNIFESP
}

\begin{abstract}
A retina humana ainda não está totalmente desenvolvida no nascimento. Só após o nascimento é que ocorrem mudanças anatômicas como o aumento na densidade de cones centrais e o alongamento do segmento externo dos fotorreceptores. As mudanças funcionais que ocorrem na retina com a maturação no primeiro ano de vida podem ser avaliadas pela técnica do eletrorretinograma de campo total, que representa a atividade somada da retina distal em resposta à luz. Abordaremos aspectos da maturação funcional da retina avaliada pelo eletrorretinograma em bebês prematuros.
\end{abstract}

Descritores: Visão. Retina. Recém-nascidos prematuros.

$\mathrm{O}$ rocesso visual inicia-se nos fotorreceptores, onde a luz é absorvida e um sinal neural é gerado. Esse processo de transformação de energia luminosa em sinal elétrico numa forma comunicável de energia química é chamado de fototransdução. Nas últimas décadas, uma série de eventos ligada ao processo de fototransdução foi descrita e melhor compreendida. Os fotorreceptores são cerca de 125 milhões e estão localizados em uma camada específica da retina, transformando o padrão de luz e sombra da imagem retiniana em padrão correspondente de gradações na imagem neural. A partir de seus fotopigmentos, os fotorreceptores começam o processo de dar cor ao mundo.

A retina humana é ainda imatura no nascimento. Várias mudanças anatômicas ocorrem só depois, como o aumento na densidade de cones cen-

1 Docente Adjunta, Doutora em Ciências, UNIFESP-Escola Paulista de Medicina, Departamento de Oftalmologia, Laboratório de Eletrofisiologia Visual Clínica. Endereço eletrônico: aberezovsky@oftalmo.epm.br 


\section{Adriana Berezovsky}

trais e o alongamento do segmento externo dos fotorreceptores. As mudanças funcionais, que ocorrem na retina durante o processo de maturação podem ser avaliadas com o eletrorretinograma de campo total (ERG), que representa a atividade somada da retina distal em resposta à luz.

\section{ERG na avaliação da função retiniana}

Os primeiros sinais elétricos de um órgão sensorial foram registrados no olho, por Holmgren em 1865 (citado por De Rouck, 2006) e, independentemente, por DeWar e McKendrik em 1871 (citados por De Rouck, 2006), ambos os grupos estimulando o olho intacto com lampejos de luz e registrando a resposta elétrica em galvanômetros comuns (lentos e de baixa sensibilidade). Esses registros correspondentes à resposta de massa da retina foram chamados de potenciais de ação retinianos, em analogia aos potenciais de ação nervosos e musculares, e passaram a ser designados como eletrorretinograma (ERG), como são ainda hoje conhecidos (De Rouck, 2006).

O ERG é um registro complexo de potenciais elétricos originários da retina em resposta à estimulação luminosa. É registrado em humanos, a partir da superfície da córnea, geralmente por meio de um eletrodo de lente de contato. A onda elétrica registrada corresponde à diferença de potencial entre esse eletrodo e um segundo eletrodo de referência, que pode estar na própria lente de contato (lente bipolar) ou em qualquer lugar sobre a pele, desde que próximo ao eletrodo ativo (lente monopolar). O ERG obtido com um flash curto de luz de intensidade moderada conta com um componente inicial córneo-negativo chamado onda- $a$, que reflete a atividade de transdução dos fotorreceptores, tanto dos bastonetes como dos cones. A onda- $a$ é seguida por componentes que surgem de processos pós-receptores, atingindo seu pico a aproximadamente 20 milissegundos (ms) após a apresentação do estímulo. O primeiro destes é a onda- $b$, um potencial córneo-positivo que, no olho de mamíferos, alcança seu pico de 60 a 100 ms após a apresentação de um flash de luz de intensidade moderada, sobre uma ampla faixa de condições de estimulação, excedendo em muito a amplitude da onda- $a$ 


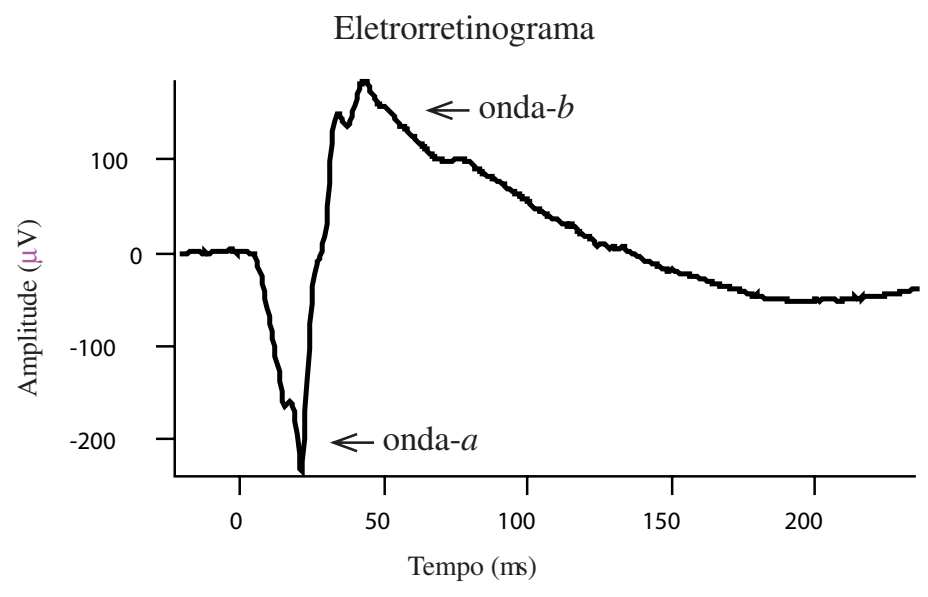

Figura 1. Eletrorretinograma de campo total de um adulto normal em resposta a um estímulo luminoso de máxima intensidade.

As ondas $a$ e $b$ ocorrem durante a apresentação do estímulo, desde que seja suficientemente longo.

\section{Separação dos componentes de cones e bastonetes}

Dados psicofísicos sobre sensibilidade espectral dos sistemas de cones e bastonetes revelam que os bastonetes são mais sensíveis do que os cones em todo o espectro visível, exceto no extremo de comprimentos de onda longos do espectro, em que há progressiva diminuição dessa diferença. Na região dos comprimentos de onda do extremo visível do lado vermelho $(\lambda>680 \mathrm{~nm})$, não há absorção de luz pelos bastonetes, podendo-se, portanto, isolar a função dos cones. A contribuição dos bastonetes ao ERG pode ser isolada se for registrada no estado adaptado ao escuro, com estímulos de baixa intensidade, no extremo oposto do espectro visível, ou seja, na região de comprimentos de onda curtos (azul $-\lambda<450 \mathrm{~nm}$ ). Dadas as diferenças de sensibilidade entre os dois sistemas, estímulos de baixa intensidade de comprimento de onda longo (vermelho-alaranjado, $\lambda=580 \mathrm{~nm}$ ) também podem ser utilizados para estimular apenas os bastonetes. 


\section{Adriana Berezovsky}

Uma forma clinicamente mais eficiente de isolar os dois componentes é através de estímulos luminosos intermitentes ("flicker"), onde a resolução temporal permite distinguir o sistema dos cones do de bastonetes. Os bastonetes não acompanham frequiências de estimulação acima de $25 \mathrm{~Hz}$, enquanto os cones acompanham freqüências até $60 \mathrm{~Hz}$ e são pouco sensíveis às freqüências baixas. Assim, com estimulação de baixa freqüência, obtém-se a resposta acromática ou de luminância dos bastonetes. Frequiências relativamente altas $(25 \mathrm{a} 30 \mathrm{~Hz})$ isolam a resposta dos cones, revelando uma curva de sensibilidade espectral com três picos, correspondentes a cada um dos tipos de fotorreceptores.

\section{Protocolo-padrão do eletrorretinograma}

Em 1989, a International Society for Clinical Electrophysiology of Vision (ISCEV), juntamente com a Foundation Fighting Blindness, recomendaram um protocolo-padrão internacional para o uso clínico do ERG de campo total (full-field ERG). A finalidade desse protocolo foi de que um registro eletrorretinográfico feito em qualquer clínica ou laboratório de pesquisa do mundo pudesse ser interpretado e analisado de modo uniforme. Visto que há no mercado diferentes opções de equipamentos e que os eletrodos usados podem ser de tipos diferentes, a ISCEV recomenda que cada laboratório ou serviço clínico de eletrofisiologia visual desenvolva sua própria padronização, examinando um grupo de voluntários normais. No Brasil, já existem estudos normativos para padronização do ERG de acordo com o protocolo-padrão da ISCEV. Os parâmetros a serem usados nesse protocolo referem-se a amplitude pico-a-pico, que é definida em microvolts $(\mu \mathrm{V})$ como o tamanho da resposta entre o pico da onda- $a$ e o pico da onda- $b$, e tempo de culminação da onda- $b$, que é definido em milissegundos como o tempo entre o estímulo e o pico da resposta da onda- $b$. O protocolo-padrão tem cinco diferentes componentes, que devem ser registrados após 30 minutos de adaptação do olho ao escuro, com a pupila completamente dilatada:

1. Resposta de bastonetes

2. Resposta escotópica máxima 


\section{Maturação Funcional da Retina em Bebês Prematuros}

\section{Potenciais oscilatórios}

4. Resposta fotópica de cones

5. Estímulo de "flicker"

Resposta de bastonetes - É a primeira resposta a ser registrada, após 30 minutos de adaptação ao escuro, com estímulo de flash de luz branca com sua intensidade máxima atenuada em 2,5 unidades logarítmicas, utilizandose filtros de densidade neutra. Esse nível de iluminação, abaixo do limiar de resposta dos cones, produz apenas respostas de bastonetes. A forma da onda, nesse tipo de resposta, em sujeitos normais, consiste de uma onda- $a$ córneonegativa menor do que $20 \mu \mathrm{V}$ e uma onda- $b$ córneo-positiva, lenta, atingindo sua amplitude máxima em torno de $80 \mathrm{~ms}$.

Resposta de bastonetes
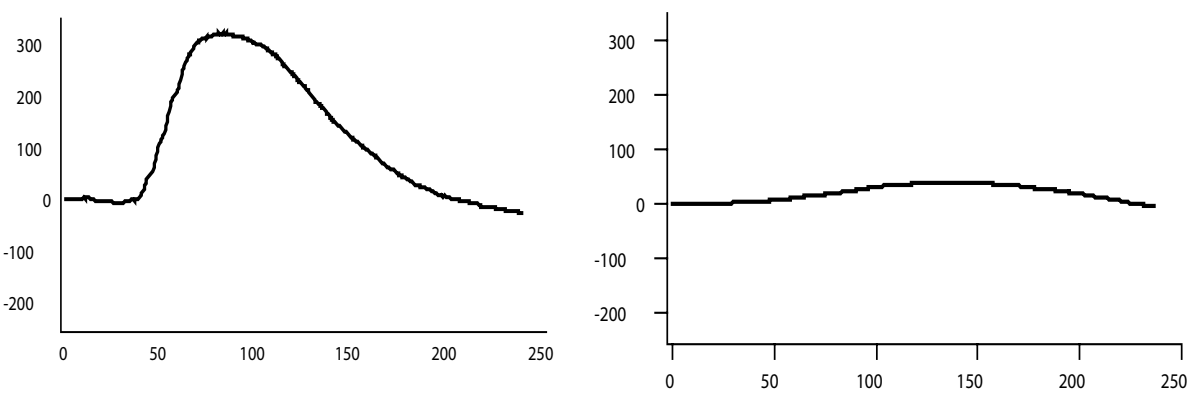

Tempo (ms)

Figura 2. Resposta de bastonetes de um adulto normal (painel à esquerda) e de um bebê normal de cinco semanas (painel à direita) em resposta a um estímulo de máxima intensidade atenuada em 2,5 unidades logarítmicas. Registros colhidos sob consentimento livre e esclarecidos no Laboratório de Eletrofisiologia Visual Clínica da UNIFESP (Escola Paulista de Medicina).

Resposta escotópica máxima - É obtida com estímulos de alta intensidade e contém tanto as respostas de cones como a de bastonetes. A estimulação é feita no estado adaptado ao escuro e sem a presença de luz de fundo, com intervalo de no mínimo 10 segundos (s) entre cada estimulação, devido à alta intensidade do estímulo. 


\section{Adriana Berezovsky}

Resposta escotópica máxima

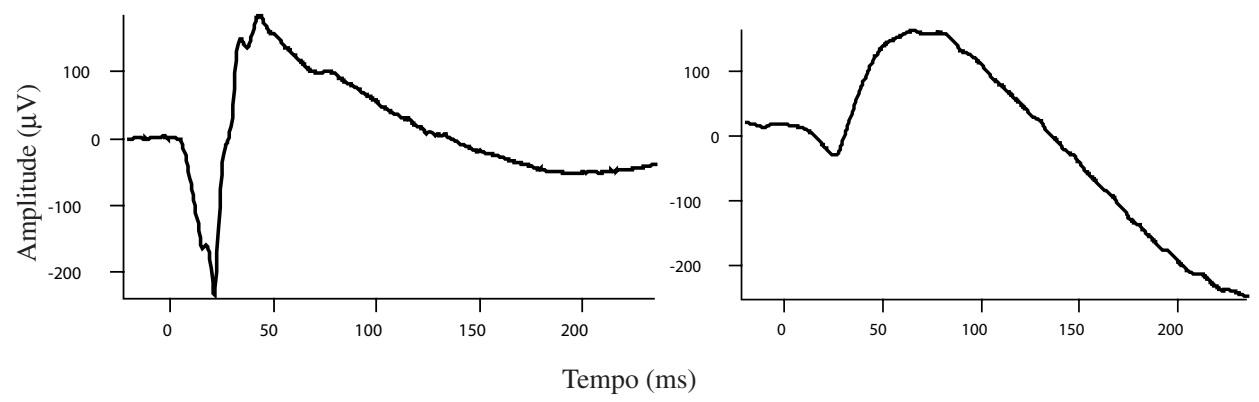

Figura 3. Resposta escotópica máxima de um adulto normal (painel à esquerda) e de um bebê normal de cinco semanas (painel à direita) em resposta a estímulo de máxima intensidade. Registros colhidos sob consentimento livre e esclarecidos no Laboratório de Eletrofisiologia Visual Clínica da UNIFESP (Escola Paulista de Medicina).

Potenciais oscilatórios - Os potenciais oscilatórios também são obtidos com o olho adaptado ao escuro. A intensidade do estímulo é a mesma da resposta máxima, porém é colhida com filtro passa-alto em $100 \mathrm{~Hz}$. Os potenciais aparecem na porção ascendente da resposta máxima e refletem a atividade das camadas internas da retina.

Potenciais oscilatórios

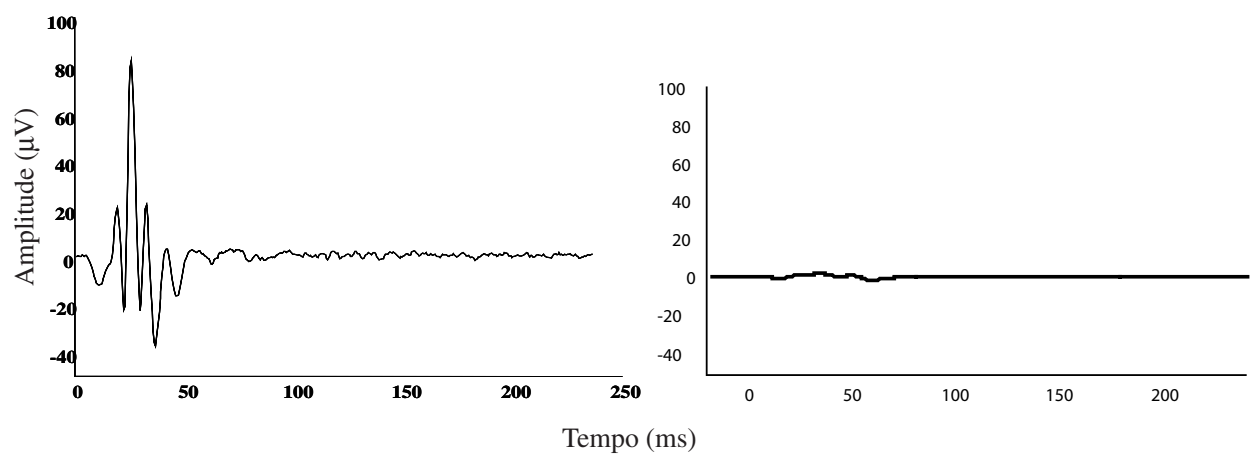

Figura 4. Potenciais oscilatórios de um adulto normal em resposta a estímulo de máxima intensidade (painel à esquerda) e de um bebênormal de cinco semanas (painel à direita) em resposta a estímulo de máxima intensidade. Registros colhidos sob consentimento livre e esclarecidos no Laboratório de Eletrofisiologia Visual Clínica da UNIFESP (Escola Paulista de Medicina). 
Resposta fotópica de cones a flash isolado - Reflete somente a atividade dos cones. É colhida saturando-se os bastonetes através de uma luz branca de fundo com luminância de aproximadamente $34 \mathrm{~cd} / \mathrm{m}^{2}$. É necessário um período de adaptação à luz de 10 minutos (min), pois as respostas dos cones tendem a crescer nos primeiros minutos. A intensidade do estímulo luminoso é máxima.

Respostas fotópica de cores

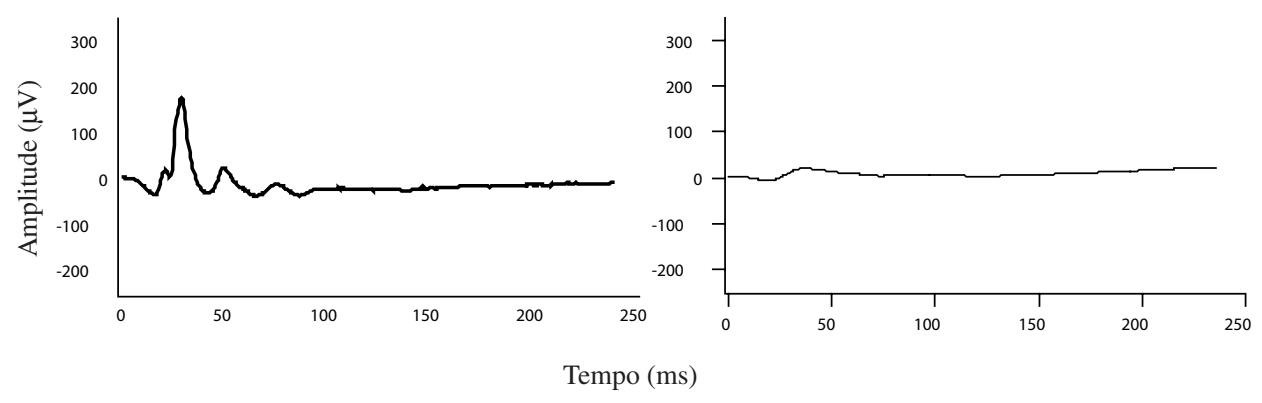

Figura 5. Resposta fotópica de cones de um adulto normal (painel à esquerda) e de um bebê normal de cinco semanas (painel à direita) em resposta a estímulo de máxima intensidade com luz branca de fundo. Registros colhidos sob consentimento livre e esclarecidos no Laboratório de Eletrofisiologia Visual Clínica da UNIFESP-Escola Paulista de Medicina).

Estímulos "flicker" - A resposta à estimulação intermitente em uma frequiência de $30 \mathrm{~Hz}$ (60 reversões entre claro e escuro/segundo) é registrada na presença da luz de fundo $\left(34 \mathrm{~cd} / \mathrm{m}^{2}\right)$, após um período de adaptação à luz de $10 \mathrm{~min}$, isolando-se as respostas dos cones. A amplitude, ou seja, o tamanho da resposta é medido do pico da resposta mínima ao pico da resposta máxima. O tempo de culminação da onda- $b$ é medido a partir do início do estímulo até o pico máximo da onda- $b$. 


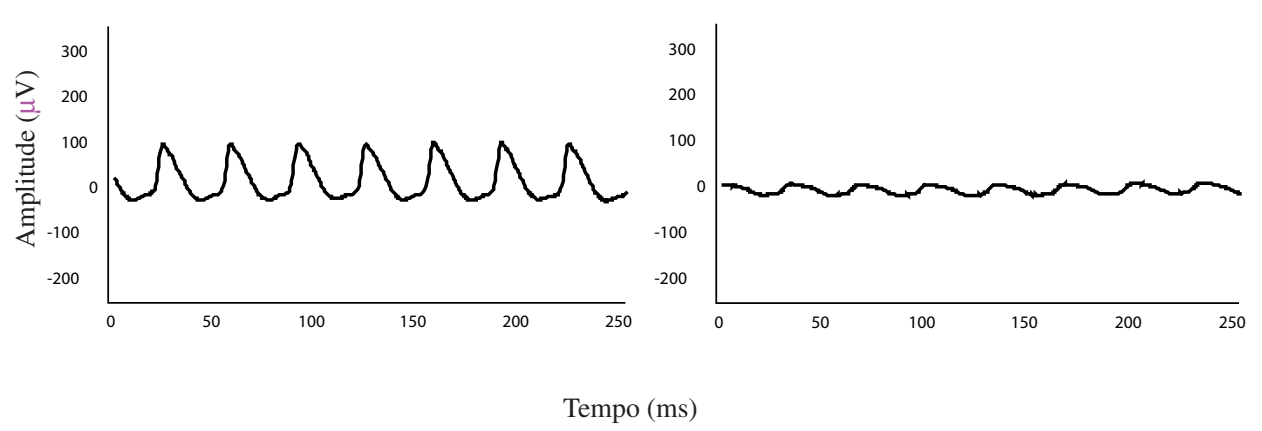

Figura 6. Resposta de um adulto normal (painel à esquerda) e de um bebê normal de cinco semanas (painel à direita) em resposta a estímulos intermitentes ("flicker") com luz branca de fundo. Registros colhidos sob consentimento livre e esclarecidos no Laboratório de Eletrofisiologia Visual Clínica da UNIFESP - EPM.

\section{ERG em bebês prematuros}

A taxa de sobrevida dos prematuros nascidos com baixo peso tem aumentado consideravelmente nos últimos anos e, nesse período, alguns fatores podem afetar o desenvolvimento normal da visão, levando à chamada retinopatia da prematuridade, uma doença proliferativa vascular da retina, relacionada ao peso, às condições de nascimento e à idade gestacional. Quanto mais baixo o peso e menor a idade gestacional, maiores as chances de o bebê prematuro desenvolver a retinopatia da prematuridade. $\mathrm{O}$ diagnóstico preciso e o tratamento adequado são fundamentais para evitar cegueira. Um aspecto importante dentre os causadores da doença é a imaturidade da retina nesses bebês.

As mudanças funcionais que ocorrem na retina com a maturação podem ser avaliadas nos bebês com o ERG de campo total, pois ele representa a atividade somada da retina distal em resposta à luz. O ERG também tem sido um instrumento de estudo, possibilitando a avaliação da influência de fatores nutricionais no desenvolvimento da retina e também os efeitos da retinopatia da prematuridade. 


\section{Maturação Funcional da Retina em Bebês Prematuros}

Em 1952, Zetterstrom demonstrou a ocorrência de atividade elétrica retiniana na presença de flash de luz em prematuros com peso a partir de $1000 \mathrm{~g}$. A atividade do ERG na primeira semana de vida ocorreu somente em bebês com peso superior a 2000g. Nos casos de baixo peso, o ERG mostrou-se presente somente a partir de 9 semanas de vida. Todos os bebês pesquisados nasceram entre 1 e 10 semanas antes do termo. Winkelman e Horsten, em 1962, detectaram respostas do ERG a luz brilhante poucas horas após o nascimento em crianças com idade de 34 semanas pós-concepção.

Mets, Smiths, Pokorny e Pass (1995) estudaram 13 prematuros com peso variando de 850 a $2540 \mathrm{~g}$ e idade gestacional estimada entre 27 e 36 semanas. Eles demonstraram presença da onda- $b$ e ausência da onda- $a$ no primeiro mês de vida. Aos seis meses, ambas as ondas ocorreram de forma similar ao que ocorre num adulto. Esses dados têm valor preditivo, em que a amplitude da onda- $b$ escotópica é zero com 32 semanas gestacionais, e são concordantes com Birch, Birch, Hoffman e Uauy (1992), que relataram que as respostas dos bastonetes aparecem por volta das 35-36 semanas gestacionais, com amplitudes pequenas e latência maiores do que as que ocorrem no adulto.

Berezovsky, Moraes, Nusinowitz e Salomão (2003) estudaram prematuros saudáveis divididos em dois grupos de acordo com a idade (três e oito semanas de idade corrigida). $\mathrm{O}$ estudo mostrou um efeito maturacional, evidenciando aumento da amplitude e diminuição do tempo de resposta para resposta de cones no grupo com oito semanas. A taxa de desenvolvimento da morfologia das ondas do ERG foi comparável à dos bebês nascidos a termo.

O estudo do desenvolvimento da função retiniana pelo ERG em bebês prematuros tem mostrado que estes, quando não desenvolvem a retinopatia da prematuridade, têm desenvolvimento semelhante ao dos bebês nascidos a termo. Além disso, estudos eletrorretinográficos de crianças com retinopatia da prematuridade podem favorecer a compreensão do efeito da doença e esclarecer sobre a eficácia dos protocolos de tratamento oferecidos. 


\title{
Adriana Berezovsky
}

Berezovsky, A. (2007). Retinal function maturation in preterm infants. Psicologia USP, 18(2), 35-45.

\begin{abstract}
The human retina is not fully developed at birth. Several anatomic changes occur postnatally in the developing retina including an increase in cone density and elongation of photoreceptor outer-segments. The functional changes that occur in the retina with maturation during the first year of life can be evaluated with full-field electroretinogram, which represents the summed activity of the distal retina in response to light. Functional maturation of the retina is addressed in preterm infants assessed by electroretinography.
\end{abstract}

Index terms: Vision. Retina. Premature infants.

Berezovsky,A. (2007). La maturation retinienne chez les bébés prématurés Psicologia USP, 18(2), 35-45.

Résumé: La rétine humaine n'est pas entièrement développée à la naissance. Plusieurs changements anatomiques ne se déroulent qu'après la naissance, y compris une augmentation de la densité des cônes et l'élongation du segment extérieur des photorécepteurs. Il est possible d'évaluer les changements fonctionnels de la rétine developpés pendant la première année de vie par l'electroretinogram plein champ, où on représente l'activité de la rétine distale en réponse à la lumière. Cela dit, on présentera certains aspects de la maturation fonctionnelle de la rétine chez les bébés prématurés.

Mots-clés: Vision. Rétine. Bébé prématuré.

\section{Referências}

Berezovsky, A., Moraes, N. S. B., Nusinowitz, S., \& Salomão, S. R. (2003). Standard fullfield electroretinography in healthy preterm infants. Documenta Ophthalmologica, 107, 243-249. 


\section{Maturação Funcional da Retina em Bebês Prematuros}

Birch, D. G., Birch, E. E., Hoffman, D. R., \& Uauy, R. D. (1992). Retinal development in very-low-birth-weight infants fed diets differing in omega-3 fatty acids. Investigative Ophthalmology \& Visual Science, 33, 2365-2376.

De Rouck, A. F. (2006). History of the electroretinogram. In J. R. Heckenlively \& G. B. Arden (Eds.), Principles and practice of clinical electrophysiology of vision (2nd ed., pp. 3-10), Cambridge, MA: MIT Press.

Mets, M. B., Smiths, V. C., Pokorny, J., \& Pass, A. (1995). Postnatal retinal development as measured by the electroretinogram in premature infants. Documenta Ophthalmologica, 90, 111-127.

Winkelman, J. E., \& Horsten G. P. M. (1962). The ERG of premature and full-term born infants during their first days of life. Ophthalmologica, 143, 92-101.

Zetterstrom, B. (1952). Flicker electroretinograohy in new-born infants. Acta Ophthalmologica, 33,157-166.

Recebido em: 14.07.2004

Aceito em: 7.10.2004 
